\title{
Red yeasts and carotenoid production: outlining a future for non-conventional yeasts of biotechnological interest
}

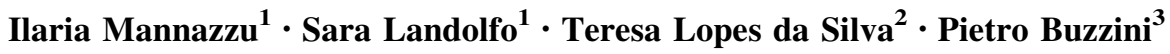

Received: 15 June 2015/ Accepted: 14 August 2015

(C) Springer Science+Business Media Dordrecht 2015

\begin{abstract}
Carotenoids are one of the most common classes of pigments that occur in nature. Due to their biological properties, they are widely used in phytomedicine and in the chemical, pharmaceutical, cosmetic, food and feed industries. Accordingly, their global market is continuously growing, and it is expected to reach about US $\$ 1.4$ billion in 2018. Carotenoids can be easily produced by chemical synthesis, although their biotechnological production is rapidly becoming an appealing alternative to the chemical route, partly due to consumer concerns against synthetic pigments. Among the yeasts, and apart from the pigmented species Phaffia rhodozyma (and its teleomorph Xanthophyllomyces dendrorhous), a handful of species of the genera Rhodosporidium, Rhodotorula, Sporobolomyces and Sporidiobolus are well known carotenoid producers. These are known as 'red yeasts', and their ability to synthesize mixtures of carotenoids from low-cost carbon sources has been broadly studied recently. Here, in agreement with the renewed interest in microbial carotenoids, the recent literature is reviewed regarding the taxonomy of the genera Rhodosporidium, Rhodotorula, Sporobolomyces and Sporidiobolus, the stress factors that influence their carotenogenesis, and the most advanced analytical tools for evaluation of carotenoid production.
\end{abstract}

Ilaria Mannazzu

imannazzu@uniss.it

1 Dipartimento di Agraria, Università degli Studi di Sassari, Viale Italia 39, Sassari, Italy

2 Unidade de Bioenergia, Laboratorio Nacional de Energia e Geologia, Estrada do Paço do Lumiar 22, Lisbon, Portugal

3 Dipartimento di Scienze Agrarie, Alimentari e Ambientali, Collezione dei Lieviti Industriali (DBVPG), Università di Perugia, Borgo XX Giugno 74, Perugia, Italy
Moreover, a synopsis of the molecular and "-omic" tools available for elucidation of the metabolic pathways of the microbial carotenoids is reported.

Keywords Basidiomycetous yeast - Carotenoid analysis . Flow cytometry $\cdot$-omic tools $\cdot$ Transformation

\section{Introduction}

Carotenoids are 40-carbon isoprenoids that are generally separated into carotenes (e.g., $\beta$-carotene) and xanthophylls (oxygenated carotenoids, such as astaxanthin), and are usually localized within intracellular lipid droplets (Buzzini et al. 2010). Owing to their bright colour, and because of a variety of their properties, carotenoids represent a valuable class of molecules for industrial applications (Johnson and Echavarri-Erasun 2011). In particular, they are widely exploited on an industrial scale as additives in pharmaceutical, chemical, food and feed products, mostly as vitamin A precursors (Johnson and Schroeder 1995; Vachali et al. 2012). Consequently, their industrial market is expected to reach about US\$1.4 billion in 2018 (BCC Research 2011).

To satisfy the increasing demand for carotenoids, they can be produced easily by chemical synthesis. However, due to general consumer concern against synthetic pigments, the biotechnological production of carotenoids (via de-novo microbial synthesis) is becoming an appealing alternative to the chemical route.

Among the microorganisms that can produce carotenoids, there are a number of basidiomycetes yeast species that grow as pigmented colonies, and are for this reason known as 'red yeasts'. Phaffia rhodozyma and its teleomorph Xanthophyllomyces dendrorhous are by far the 